\title{
Questes
}

\section{Les hommes illustres : introduction}

\section{Marion Chaigne-Legouy et Anne Salamon}

\section{(2) OpenEdition}

\section{Journals}

\section{Édition électronique}

URL : http://journals.openedition.org/questes/466

DOI : 10.4000/questes.466

ISSN : 2109-9472

\section{Éditeur}

Les Amis de Questes

\section{Édition imprimée}

Date de publication : 15 octobre 2009

Pagination : 5-23

ISSN : 2102-7188

\section{Référence électronique}

Marion Chaigne-Legouy et Anne Salamon, «Les hommes illustres : introduction », Questes [En ligne],

17 | 2009, mis en ligne le 01 janvier 2014, consulté le 23 septembre 2020. URL : http:// journals.openedition.org/questes/466 ; DOI : https://doi.org/10.4000/questes.466 


\section{Les Hommes illustres Introduction}

\section{Marion Chaigne et Anne SALAMON}

Le thème des Hommes illustres trouve son origine dans les textes antiques du même nom, De viris illustribus. Il permet par conséquent d'examiner de façon privilégiée les rapports qu'entretiennent le Moyen Âge, puis la Renaissance, avec l'héritage antique, en suivant la transmission d'un genre dans une combinaison de continuité et de changement, continuité de formes et modèles anciens, associés à de nouveaux usages et de nouvelles conceptions, avant la Renaissance et son « retour » à l'Antiquité. Plus que d'une réflexion générale sur la notion de « grand homme», c'est de la pratique antique et de ses spécificités que nous voudrions partir.

La biographie est, à ses origines, un genre polymorphe. Elle prend son essor aux $V^{\mathrm{e}}$ et $\mathrm{IV}^{\mathrm{e}}$ siècles avant Jésus Christ en Grèce, dans un contexte de développement de la recherche historique centrée sur l'histoire politique ${ }^{1}$. La logique du panégyrique, de l'exemplarité y est prédominante. À Rome, la biographie a partie liée avec les pratiques funèbres et celles du culte des ancêtres, le mos majorum, illustré par de grandes figures, les exempla majorum. Caton explique ainsi que les mérites des Hommes illustres étaient loués lors des banquets ${ }^{2}$. Les listes des De viris illustribus en tant que telles ne peuvent être constituées que de personnages illustres, c'est-à-dire étymologiquement «brillants, éclairés, en vue », d'où notre illustre, «dont le renom est très grand du fait de qualités, de mérites extraordinaires ou d'actions exceptionnelles qui s'y attachent ». La gloire et

\footnotetext{
${ }^{1}$ Élizabeth GAUCHER, La Biographie Chevaleresque. Typologie d'un genre (XIII $-X V^{e}$ siècle), Paris, Champion, 1994.

${ }^{2}$ C'est Cicéron qui signale ce fait dans les Tusculanes (IV, 3).
} 
la renommée sont au cœur de la problématique des Hommes illustres et y sont déclinées dans une série de manifestations individuelles.

Si l'attention au cadre spatio-temporel s'impose dans toute étude sur le thème des Hommes illustres, il s'agit aussi d'appréhender l'ensemble de ses supports, de la suite de biographies à la simple liste de noms dans sa forme littéraire, des miniatures aux suites de portraits dans les arts visuels. Il faudra donc s'interroger sur la signification de la mise en série des biographies : une tension parcourt les catalogues d'Hommes illustres, entre l'évocation d'une existence individuelle et l'illustration d'un système de valeurs émergeant de la confrontation de ces vies. À travers le personnage décrit apparaît en filigrane toute une définition et une conception de l'homme. En effet, toute biographie, dans la mesure où elle implique la réorganisation d'une existence particulière et des choix, est le véhicule d'une idéologie ou du moins d'une certaine conception de l'histoire et de l'individu. Il sera intéressant dans ce contexte de réfléchir à l'émergence de la notion d'individu à la fin du Moyen Âge.

Les caractéristiques formelles et idéologiques des recueils de biographies de l'Antiquité peuvent servir de points de comparaison pour éclairer leurs avatars médiévaux et renaissants. Si les Vies Parallèles de Plutarque sont la première œuvre à laquelle on pense lorsqu'on évoque les Hommes illustres, elles ont été presque complètement ignorées de la période médiévale et n'ont commencé à véritablement circuler et susciter un large intérêt qu'au $\mathrm{XV}^{\mathrm{e}}$ siècle en Italie ${ }^{3}$. En France, elles ne deviennent les Vies des hommes illustres grecs et romains comparées l'une avec l'autre que sous la plume de Jacques Amyot qui traduit le texte pour Henri II, en 1559. En accordant une attention particulière à l'originalité des

\footnotetext{
${ }^{3}$ Roberto WeIss, Medieval and Humanist Greek, Collected Essays, Padoue, Antenor, 1977, p. 204-217, et Marianne PADE, The Reception of Plutarch's Lives in FifteenthCentury Italy, Copenhague, Museum Tusculanum Press, 2007.
} 
différentes aires linguistiques utiles à cette étude, notamment française et italienne, il s'agira d'examiner la postérité du genre De viris illustribus et la réflexion sur la gloire qu'il suscite. Avant de se pencher sur la différentiation spatio-temporelle des usages des Hommes illustres, il convient d'aborder, autour de la naissance et de l'implantation du christianisme, ses réappropriations dans une christianitas comprise comme une institution globale encadrant l'ensemble de la société.

\section{La rupture du christianisme et les réappropriations d'un genre littéraire}

\section{L'influence de saint Jérôme: l'apparition des recueils biobibliographiques ${ }^{4}$}

Saint Jérôme compose à la fin du $\mathrm{IV}^{\mathrm{e}}$ siècle un De viris illustribus, comportant cent trente-cinq brèves notices biobibliographiques d'auteurs chrétiens, qui connaît une très grande diffusion au Moyen Âge. Son projet correspond à une prise de position idéologique forte puisqu'il s'agit de prouver aux païens que les chrétiens bénéficient, eux aussi, d'une bonne littérature et de grands auteurs. Cette œuvre est à l'origine de deux changements majeurs que connait le genre au Moyen Âge : une focalisation sur l'appartenance de ses membres à la Chrétienté et une spécialisation de l'écriture en un catalogue d'auteurs, héritée du De viris illustribus ${ }^{5}$ de Suétone.

Le texte de saint Jérôme est d'abord complété par Gennadius de Marseille puis par Isidore de Séville ( $\mathrm{V}^{\mathrm{e}}$ et $\mathrm{VI}^{\mathrm{e}}$ siècles). Les manuscrits

\footnotetext{
${ }^{4}$ Cf. Mary A. Rouse et Richard H. Rouse, « Bibliography before print : the medieval De viris illustribus ", in Peter GANZ (éd.), The Role of the Book in Medieval Culture. Proceedings of the Oxford International Symposium 29 septembre-1 ${ }^{\text {er }}$ octobre 1982, Bibliologia. Elementa ad Librorum Studia Pertinentia, Turnouht, Brepols, 1986, p. 133153.

${ }^{5}$ Contrairement à la Vie des douze Césars, le De viris illustribus de Suétone a été presque entièrement perdu. Nous savons cependant qu'il était constitué de cinq parties, consacrées aux poètes, orateurs, historiens, philosophes et grammairiens.
} 
confirment que ces trois textes formaient un corpus complémentaire qui a servi de bibliographie historique durant toute la période médiévale. D'un point de vue encyclopédique, les notices de Jérôme servent d'introductions aux œuvres copiées et de liste de référence pour compléter les fonds de patristique. Ces pratiques se poursuivent tout au long du Moyen Âge avec l'élaboration de nouvelles compilations comme celle de Sigebert de Gembloux au $\mathrm{XI}^{\mathrm{e}}$ siècle. Par la suite, les recueils se constituent autour d'une spécialisation par zone géographique ou par ordre monastique, traduisant la nécessité locale de "posséder » ses propres grands hommes. Interroger le genre des De viris illustribus à partir d'une contextualisation, ici le développement d'un monachisme varié et la mise en place des principautés, peut ainsi s'avérer fécond.

Virtus et exemplarité au cœur d'une production littéraire et iconographique chrétienne

La première évolution remarquable entre les auteurs païens et chrétiens réside dans le sujet même de leurs écrits. Le délaissement des généraux et des orateurs investis dans la vie de la cité au profit des écrivains chrétiens n'enlève en rien au public de ces ouvrages le goût des hommes exemplaires. Ces derniers se définissent par leur manière de résister aux assauts de la Fortune ou de l'Envie, dans leur façon de surmonter l'adversité. Tous possèdent cette virtus, cette qualité discriminante qui détermine l'appartenance ou non à la série des Hommes illustres.

La virtus est une condition nécessaire pour être qualifié d'illustris, mais sa définition n'est pas monolithique. Les variations de sa conception permettent d'élargir le répertoire des hommes sélectionnés. Première des quatre vertus cardinales antiques, la virtus se définit tout d'abord comme le courage physique du soldat sur le champ de bataille avant de s'infléchir, 
sous l'influence de la pensée stoïcienne, dans le sens d'un courage moral nécessaire à l'homme pour accéder à la sagesse. Une énergie individuelle caractérise donc la virtus. Les chrétiens d'exception, les saints, la détiennent également : en contrepartie de leurs mérites, ils sont gratifiés par Dieu d'une force (virtus) qui demeure présente et continue d'agir, après leur décès, par le truchement de leur dépouille. Cette acception est élargie dès l'époque mérovingienne au sens de force, puissance ou capacité à réaliser des miracles ${ }^{6}$. La virtus, qui lie l'ici-bas et l'au-delà, justifie le développement du culte des saints, intercesseurs entre les deux mondes. La question se pose alors des rapports entre un ancien genre littéraire défini, célébrant des viri illustres distingués par leurs qualités civiques ou militaires, et l'apparition d'une forme littéraire de célébration de personnages loués pour leur foi et, le plus souvent, pour leur appartenance à la vie ecclésiastique.

Le culte des saints a conduit à la constitution de martyrologes - des listes de saints disposés selon l'ordre du calendrier liturgique - puis de collections de Vies de saints à partir du VIII ${ }^{\mathrm{e}}$ siècle. Connaissant leur apogée au XIII ${ }^{\mathrm{e}}$ siècle, ces regroupements biographiques utilisent la composition-type du recueil de viri illustres. La variété des légendiers repose sur la diversité de leur usage. Le plus connu d'entre eux, La Légende dorée de Jacques de Voragine, est un abrégé apparu dans la mouvance des ordres mendiants afin de servir leur mission de prédicateurs $^{7}$. Son emploi s'est élargi aux lectures privées laïques et religieuses, comme le montrent les traductions en langues vernaculaires et la richesse de certains exemplaires. Fréquemment, des miniatures scandent

\footnotetext{
${ }^{6}$ André VAucheZ, La Sainteté en Occident aux derniers siècles du Moyen Âge d'après les procès de canonisation et les documents hagiographiques, Rome, École française de Rome, 1981, p. 497.

${ }^{7}$ Nous renvoyons à la riche introduction d'Alain BOUREAU in JACQUES DE VorAGINE, La Légende dorée, Alain Boureau (éd.), Paris, Gallimard, "Bibliothèque de la Pléiade », 2004.
} 
les vitae de ces recueils hagiographiques et s'attardent sur les attributs de la virtus du saint. Les représentations les plus nombreuses illustrent le courage moral des martyrs devant les souffrances infligées par le bourreau, à l'origine du miracle de leur survie ${ }^{8}$. Une double dimension - individuelle (une miniature ouvrant chaque vie) et collective (hiératisme des figures) se reflète dans une iconographie qui vise surtout à illustrer un idéal de la sainteté proposant un nouveau modèle d'exemplarité.

La tension entre l'individualisation et la formalisation est un fil conducteur permanent des vies des Hommes illustres. L'héroïsme naît du dévouement absolu de son sujet à une cause, la patrie durant l'Antiquité, le triomphe du christianisme dès le début du Moyen Âge. Cette grandeur s'incarne dans la production des De viris illustribus au travers de figures concrètes, inscrites dans des imagines, dont le regroupement collectif doit faire naître un sentiment d'admiration et d'émulation, quel que soit le domaine où leurs qualités s'exercent".

Une réception active est attendue de la part du public : l'exemple de ces existences individuelles fournit un modèle bénéfique à l'amélioration de la vie de chaque lecteur. Plutarque s'exprime ainsi : «l'histoire des grands hommes est comme un miroir que je regarde pour tâcher en quelque mesure de régler ma vie et de la conformer à l'image de leurs vertus, [...] d'écarter ce que la fréquentation des hommes nous apporte nécessairement de mauvais et de bas $»^{10}$. Même si l'œuvre de Plutarque est alors oubliée, la correspondance morale qu'il établit entre un pôle positif et un pôle négatif perdure dans les ouvrages médiévaux.

\footnotetext{
${ }^{8}$ Voir par exemple les illustrations, ibid., p. LXXII-LXXIII.

${ }^{9}$ Sur l'évolution de l'exemplarité à travers le temps, voir Claude BREMONT, Jacques LE GofF et Jean-Claude SCHMITT, L'Exemplum, Brepols, Turnhout, 1982, p. 43-68.

10 Plutarque, «Vie de Timoléon », in Les Vies parallèles, Robert Flaceliere et Emile CHAMBRY (trad. et éd.), Paris, Les Belles Lettres, 1, 1-1, 5.
} 
La régulation des comportements du lectorat demeure l'objectif d'une didactique scindée entre exemple et contre-exemple, reposant tous deux sur des topoi compréhensibles pour tous. Ceci est particulièrement visible dans les exempla irriguant la production hagiographique, qui se distinguent de leur usage gréco-romain : consacrés tant aux actions incarnant un idéal de sainteté qu'à celles recevant un châtiment divin mérité, ils servent «un système déjà constitué alors que l'exemplum antique le fixe dans son énoncé même ${ }^{11}$. Il faut également souligner que ces historiettes, insérées dans des récits consacrés à la célébration des archétypes de la perfection chrétienne, érigés comme modèle, traduisent la volonté de dépasser l'antagonisme entre le souci de l'éclat individuel et le salut chrétien qui repose sur une humilité remémorée aux lecteurs par leur intermédiaire. Ce jeu d'écriture ne remet pas en cause la centralité d'une exemplarité qui découle d'une gloire reconnue: gloire terrestre pour les hommes de l'Antiquité, gloire céleste pour les saints. Avec Pétrarque, une rupture majeure intervient : les temps anciens deviennent un temps exemplaire à retrouver. Dès lors, le genre des Hommes illustres est réactivé hors du cadre hagiographique.

11 Manfref FuhrmanN, «Das Exemplum in der antiken Rhetorik » in Reinhart KosellecK et Wolf-Dieter STEMPEL (éd.), Geschichte. Ereignis und Erzählung, Poetik und Hermeneutik, 5, Munich, 1973, p. 451-452. Cet article est cité dans la présentation de Jean-Michel DAVID de l'ouvrage collectif Rhétorique et histoire. L'exemplum et le modèle de comportement dans le discours antique et médiévale publié par les Mélanges de l'École française de Rome, 92 (1980), p. 13. 


\section{Les Hommes illustres, un rapport à l'histoire et à la mémoire}

De l'héritage troyen au rôle fondamental de Pétrarque et des premiers humanistes

Dans une contribution fondatrice ${ }^{12}$, Maria Monica Donato montre combien l'innovation pétrarquienne repose sur une nouvelle conscience littéraire, qui ne renie en rien le pouvoir des images, et sur une conception nouvelle de l'histoire du monde, scandée par la mise en place d'une hiérarchie de valeurs entre les époques - conception opposée à la continuité de l'histoire relevant de la Providence. Pétrarque concentre son échantillon d'Hommes illustres, dont il entreprend la rédaction des vies à partir des années 1340 , sur les périodes royale et républicaine de Rome, qu'il oppose aux âges obscurs dans lesquels il estime vivre ${ }^{13}$. Raconter l'histoire devient pour lui un moyen de transformer l'horizon de l'exemplarité profane. Dispensée en séquences chronologiques de biographies, l'histoire n'incite pas à une réflexion sur les événements, mais sur la virtus des hommes sélectionnés. Connaître pro irritare animos et donc permettre l'imitation, telle est son ambition.

La démarche de Pétrarque joue un rôle fondateur dans un triple registre : la redécouverte de l'Antiquité, une nouvelle culture de la gloire « temporelle», et la conscience du rôle de l'écrivain. Diffusées dans le contexte particulier de la péninsule italienne, ces innovations permettent au genre des Hommes illustres de connaître un nouvel essor. Il convient alors de s'interroger sur la soumission du matériel littéraire classique à de nouvelles structures artistiques apparues dans les deux derniers siècles du Moyen Âge.

\footnotetext{
12 Maria Monica DonATO, «Gli eroi romani tra storia ed "exemplum". I primi cicli umanistici », in Salvatore SETTIS (éd.), Memoria dell'antico nell'arte italiana, vol. II : I generi e i temi ritrovati, Turin, Giulio Einaudi editore, 1985, p. 95-152.

${ }^{13}$ Pétrarque désigne la période allant de la chute de l'Empire romain jusqu'à son époque par le terme de tenebrae dans une lettre à Agapito Colonno datée de 1359 (Rerum Familiarum, XX, 8).
} 
L'idée d'une perte totale de la mémoire antique durant le Moyen Âge est battue en brèche depuis des décennies par la recherche. Un souvenir des temps passés s'est toujours maintenu dans la conscience collective. En témoigne l'importance du cycle troyen développé en premier lieu en France et en Angleterre. Reposant sur une glorification des Troyens aux dépens des Grecs, cette série de héros peut être considérée, aux côtés de l'hagiographie, comme un jalon dans l'évolution et la réappropriation médiévales des De viris illustribus. Cette interprétation repose sur la rupture de ce cycle avec la perspective classique de l'historiographie romaine, célébrant avant tout le survivant Énée, et sur la mise en série des Troyens, assimilés à des victimes dont la bravoure s'est heurtée à la trahison. Ces changements substantiels sont particulièrement visibles dans l'iconographie où, en outre, ces personnages historiques sont traités sur un mode chevaleresque comme le montrent les illustrations sélectionnées par Hugo Buchtal ${ }^{14}$. L'intrication de continuités et de ruptures formelles pose avec acuité la question du degré de filiation des illustres et des héros troyens. Étudier la place qu'occupent ces derniers dans la renaissance du genre des Hommes illustres en Italie, alors qu'ils s'y diffusent à la fin du XIII $^{\text {e }}$ siècle avec l'œuvre de Guido de Columnis ${ }^{15}$, offre un axe de recherche stimulant. De l'œuvre de ce juriste de la cour de l'empereur Frédéric II jusqu'à La Divine Comédie de Dante, les Troyens sont célébrés. Ces louanges perdurent avec l'« historicisation politique » du thème des Hommes illustres et son inscription dans présent. Ces nouvelles pratiques émergent des particularités politiques et culturelles de la péninsule italienne

\footnotetext{
${ }^{14}$ Hugo Buchthal, Historia Troiana. Studies in the History of Medieval Secular Illustration, Londres, Warburg Institute, 1971.

15 Ibid., p. 5-7. L'auteur souligne la dette de cette Historia destructionis Troiae tant envers certains auteurs grecs du $\mathrm{I}^{\mathrm{er}}$ siècle, Dares Phrygius (De excidio Troiae) et Dictys Cretensis (Ephemeris de bello Troiano), qu'envers Le Roman de Troie de Benoît de Sainte-Maure, produit typique de la littérature chevaleresque du nord du royaume de France.
} 
et de la «mise en place d'une nouvelle relation à la durée » à la fin du Moyen Âge ${ }^{16}$.

Entité géographique cohérente, morcelée politiquement, l'Italie impose son unité face aux grandes puissances étrangères - notamment au royaume de France et à l'Empire germanique - par la gloire de son passé romain. Pétrarque, après avoir parcouru différentes contrées européennes, estime que «plus il voyage, plus grande est l'admiration [qu'il éprouve] pour [sa] terre natale ». Et il ajoute : «Personne n'ira nier, je crois, que la race italienne est bien plus illustre que la grecque ${ }^{17}$. Ce renouvellement de la pensée historique s'épanouit avec les humanistes et s'accompagne d'un mouvement de «recherche des Antiquités» (manuscrits, objets et témoignages archéologiques romains) - et de vastes entreprises de traduction, telles les Vies parallèles de Plutarque par Leonardo Bruni. Dans sa préface, rédigée à l'intention de Coluccio Salutati entre 1405-1406, il déplore que tant d'écrits ont été perdu « que nous n'avons retenu ni les faits ni même les noms de nos ancêtres, grâce auxquels le nom de l'Italie a retenti avec gloire dans le monde entier ». Son projet consiste à « traduire en latin l'ensemble des biographies de Plutarque [afin de] faire renaître la mémoire et la gloire des plus grands hommes ${ }^{18}$. Leonardo Bruni conçoit l'histoire romaine comme une histoire nationale glorieuse dont il faut faire la publicité. Le thème des Hommes illustres participe ainsi au dessein «civilisateur» de l'humanisme. Bien qu'imposant le rayonnement international de la péninsule, il est paradoxalement érigé en tant que révélateur et réceptacle des divisions italiennes.

${ }^{16}$ Elisabeth Crouzet-Pavan, Renaissances italiennes, Paris, Champ Vallon, 2007, p. 19.

${ }_{17}$ Ibid., p. 31.

${ }^{18}$ Leonardo Bruni, Praefatio in Vita M. Antonii ex Plutarcho traducta, in H. BARON (éd.), Leonardo Bruni Aretino. Humanistische-Philosophische Schriften, Leipzig-Berlin, Verlag und Druck Von B.G. Teubner, 1928, p. 103-104. Nous remercions Clémence Revest de nous avoir communiqué cet extrait ainsi que sa traduction. 
L'importance de la célébration civique, dynastique et patriotique: les exemples italiens et espagnols

Les relations intenses entre littérature et arts visuels favorisent la prépondérance nouvelle du motif des Hommes illustres dans les programmes culturels de l'Italie de la Renaissance. Ces figures incarnent les vertus exaltées par les humanistes au profit des gouvernants et participent, par leur lisibilité, aux stratégies de légitimation d'individus et de groupes socio-politiques à différentes échelles.

Dans une Italie divisée entre des cités-Etats progressivement gagnées par l'implantation des Seigneuries, se met en place, parmi les héros de l'Antiquité célébrés, une dichotomie entre ceux qui relèvent de la République romaine et ceux de l'Empire. Florence récupère à son profit le thème glorieux de la Rome républicaine l'emportant sur la tyrannie : la Florentina Libertas hérite de la Romana Libertas ${ }^{19}$. À cet héritage s'ajoute l'exaltation nouvelle de citoyens fameux, notamment les poètes les plus célèbres de la ville (Pétrarque, Boccace, Dante) comme autant de témoignages des vertus propres au régime républicain de la cité ${ }^{20}$. Quelques exemples notoires attestent cet usage, tels l'insertion de contemporains dans le De casibus virorum illustrium de Boccace ou le choix des portraits d'Hommes illustres exécutés dans les années 1450 par Andrea del Castagno pour la famille Carducci, exilée de la ville suite à l'implantation durable des Médicis dans le paysage politique florentin. Ces représentations comprennent des personnages publics originaires de

${ }^{19}$ Daniel ArASSE, «La fin du Moyen Âge et la première Renaissance. Peinture et sculpture », in Philippe Morel (dir.), L'Art italien, vol. 1, Paris, Citadelles et Mazenod, 1997, p. 247-248. Pour approfondir le sujet de la République florentine, voir Quentin SkinNer, Les Fondements de la politique moderne, Paris, Albin Michel, 2001 ( 1 ère éd. 1978).

${ }^{20}$ Johannes BartuschAt, «La Vie de Dante de Leonardo Bruni », in Agnès MoRINI (éd.), Figure, figures : portraits de femmes et d'hommes célèbres, ou moins, dans la littérature italienne, Saint-Etienne, Presse de 1'Université de Saint-Etienne, 2002, p. 3550. 
Florence, comme le général Pippo Spano ou le chancelier de Jeanne $\mathrm{I}^{\mathrm{re}}$ de Naples, Niccolò Acciaiuoli.

La diversité interne au genre des Hommes illustres fournit un arsenal d'objets iconographiques et de dispositifs formels qui circulent d'un groupe social à l'autre. L'évolution des institutions communales, dans le sens d'une personnalisation du pouvoir, devient une matrice essentielle pour comprendre la variété des usages de ce thème. Les nouvelles dynasties seigneuriales, cherchant à affermir leur fragile légitimité, utilisent ce genre déjà codifié et compris par tous. Le dialogue entre le texte et l'image est précoce : il faut remarquer les liens entre les séjours de Pétrarque et la réalisation des cycles muraux d'Hommes illustres par Giotto dans les palais princiers de Naples, Milan et Padoue ${ }^{21}$, malheureusement aujourd'hui perdus. Ces fresques associent la figure du commanditaire aux personnages antiques, confondant passé et présent, incluant le prince italien parmi les plus illustres. Cette confusion caractérise aussi la production de l'humaniste Pierre Paolo Vergerio qui utilise les codes biographiques d'illustres pour rédiger un Liber de Principibus Carrariensibus et gestis eorum. Le seigneur de Padoue, Francesco Novello Da Carrara, associe ainsi à l'œuvre picturale la production littéraire pour célébrer son lignage et confirme ainsi que l'appartenance symbolique à une lignée glorieuse justifie la souveraineté revendiquée par ces nouveaux princes.

S'appuyant sur cette idée, Daniel Arasse s'attache à la compréhension du programme décoratif du studiolo de Frédéric de Montefeltre $^{22}$. Lieu de la mémoire familiale et de la relation spirituelle aux ancêtres, le cabinet de travail du nouveau duc d'Urbino devient un lieu de

\footnotetext{
${ }^{21}$ Ces localités sont à associer aux maisons respectives des Angevins, Visconti et Da Carrara.

${ }^{22}$ Daniel ArASSE, « Frédéric dans son cabinet : le studiolo d'Urbino », in Le Sujet dans le tableau. Essais d'iconographie analytique, Paris, Flammarion, 2005 (1 $1^{\mathrm{rer}}$ éd. 1997), p. 17-30.
} 
représentation politique tout autant qu'un « support et [une] manifestation du développement de la sphère intime du prince ». Substituant aux portraits des ancêtres des portraits d'Hommes illustres, Frédéric - enfant naturel s'appuie sur une conception humaniste de la «vraie noblesse » adossée à des vertus qui ne doivent plus rien à la valeur du sang. Vingt-huit portraits, associés deux par deux selon le principe de l'arbre généalogique, représentent la famille idéale du duc, qui entend s'inspirer de leur modèle pour cultiver ses propres vertus et créer une nouvelle dynastie visible à tous dans une pièce faisant également office de lieu de représentation pour les ambassades.

L'exemplarité des Hommes illustres italiens ne doit cependant pas dissimuler l'usage socio-politique qui en est fait dans d'autres contrée. Les travaux récents de Béatrice Leroy ${ }^{23}$ sur les historiographes du royaume de Castille au $\mathrm{XV}^{\mathrm{e}}$ siècle sont nécessaires pour comprendre la stratification spatio-temporelle des usages de notre thème d'étude: glorification nationale par le choix de personnalités castillanes, glorification individuelle par une écriture historique biographique, inscription consciente de cette écriture dans un héritage antique, volonté de donner à voir des modèles à suivre et des vices à fuir, importance des relations de faits notables, utilisation des gloires locales comme prétexte à une réflexion sur un pouvoir royal subissant des tourments dynastiques. L'historienne met également en évidence un processus central, à savoir l'intégration des créateurs d'Hommes illustres dans ce «club» très fermé : le premier historiographe officiel de Castille, Fernan Perez de Guzman, est introduit

\footnotetext{
${ }^{23}$ Béatrice LEROY, « La mémoire politique de la Castille au $\mathrm{XV}^{\mathrm{e}}$ siècle : une galerie des hommes illustres ", in Jean KeRHERVE et Albert RIGAUdiERE (éd.), Finances, pouvoirs et mémoires : mélanges offerts à Jean Favier, Brest, Fayard, 1999, p. 301-312; Béatrice LEROY, Histoire et politique en Castille au $X V^{e}$ siècle. Les hommes illustres de Castille de Hernando del Pugar, Limoges, PULIM, 2001.
} 
par son successeur, Hernando del Pulgar, dans sa propre rédaction à la fin du XV $\mathrm{X}^{\mathrm{e}}$ siècle.

\section{Quand les artistes deviennent des Hommes illustres, une entrée dans la Modernité?}

La problématique des Hommes illustres ne peut faire abstraction des changements sociaux affectant les producteurs d'objets culturels. Pétrarque revendique son statut d'écrivain, détenteur d'un savoir fondé sur la noblesse, non plus sur l'utilité. Son Triomphe à Rome lors d'une cérémonie au Capitole (1341) signe son entrée dans certains cycles d'Hommes illustres. Cette figure emblématique montre la nécessité de questionner les rapports entre la redécouverte des textes antiques célébrant poètes et orateurs et la constitution de ces biographies contemporaines d'artistes.

Si le mot d'artista n'existe pas à la Renaissance, il est à noter que le pouvoir politique et le pouvoir intellectuel se trouvent liés de façon nouvelle à la fin du Moyen Âge. La plus grande puissance du temps, l'Église, entretient alors des rapports davantage avec des «artisansfournisseurs » qu'avec des artistes. Au contraire, les cités et les seigneurs italiens sollicitent progressivement l'ingenium des peintres et des sculpteurs pour diffuser leur programme politique, exposer à tous leur richesse et leur puissance et faire répondre à la puissance littéraire celle de l'art. Nous avons précédemment vu l'importance du couple PétrarqueGiotto à la cour des Da Carrara, des Visconti et des Angevins de Naples. Mais au-delà de cette équation, l'artiste - s'il n'en porte pas encore le nom - revendique, au nom d'un art qui dépasse la simple mécanique, la vertu d'un travail intellectuel et esthétique qui se traduit, par exemple, par l'apparition de sa signature dans le tableau.

Dès 1456, l'historien Bartolomeo Facio, secrétaire du roi de Naples, intègre trois peintres illustres de son temps parmi ses viri illustres: Gentile 
da Fabriano, Jan Van Eyck et Roger Van der Weyden ${ }^{24}$. Il faut cependant attendre Giorgio Vasari, avec les Vies des meilleurs peintres, sculpteurs et architectes (1550), pour établir définitivement la dignité culturelle de ces personnalités. Son œuvre, structurée sur le modèle des De viris illustribus avec l'emploi récurrent de topoi, notamment le passage essentiel du dépassement du maître par l'élève, repose sur l'idée d'un progrès constant de l'art du XIII ${ }^{\mathrm{e}}$ au $\mathrm{XVI}^{\mathrm{e}}$ siècle dont Florence serait l'épicentre. Sa galerie de portraits d'artistes n'a cependant pas vocation à aplanir les caractères individuels. L'impact de cette irréductibilité de la singularité au sein d'un cadre contraignant a durablement conditionné notre perception de la Renaissance : une poignée de génies aurait permis une révolution picturale, selon une interprétation des œuvres du Trecento construite à la lumière de la maîtrise des rapports spatiaux acquise durant le Quattrocento. Créateur de mythes, Vasari permet au genre des Hommes illustres de s'inscrire dans une Modernité qui considère différemment ses artistes, tant en Italie qu'en France.

\section{Variations françaises}

Si le XVI ${ }^{\mathrm{e}}$ siècle est le siècle des Hommes illustres pour la France, comme l'a montré Patricia Eichel ${ }^{25}$, cette émergence ne peut être comprise qu'en rapport avec ce qu'il est convenu d'appeler le premier humanisme français. Il repose sur le développement des relations entre la France et l'Italie et la diffusion de la culture et de la littérature italienne aux $\mathrm{XIV}^{\mathrm{e}}$ et $\mathrm{XV}^{\mathrm{e}}$ siècles. Alors que le terme $\mathrm{d}^{\prime}$ « illustre » ne commence à être utilisé qu'au $\mathrm{XV}^{\mathrm{e}}$ siècle, quelle réflexion mène-t-on à la même époque en France sur les grans ou nobles hommes?

\footnotetext{
${ }^{24}$ Daniel ArASSE, Le Sujet dans le tableau. Essais d'iconographie analytique, op. cit., 1997, p. 172.

${ }^{25}$ Patricia EICHEL-LojKIne, Le Siècle des Grands Hommes. Les recueils de Vies d'hommes illustres avec portraits du XVI siècle, Louvain, Peeters, 2001.
} 


\section{L'influence italienne en France}

La question de la réception du thème des Hommes illustres et du rapport aux auctoritates est double pour la France. Il faut en effet prendre en compte l'influence croissante des œuvres antiques, mais aussi celle des œuvres italiennes ${ }^{26}$.

Si l'influence du De viris illustribus de Pétrarque y semble peu importante à cette époque et bien moindre que celle de sa poésie, il n'en va pas de même des œuvres de Boccace. Son De casibus virorum illustrium est la première de ses œuvres à être traduite en français. Laurent de Premierfait en fournit deux versions au début du $\mathrm{XV}^{\mathrm{e}}$ siècle, en 1400 puis en 1409. Cette deuxième version, dédiée au duc de Berry, augmentée par rapport à l'original et accompagnée d'un vaste programme iconographique, a connu un immense succès ${ }^{27}$. Dans cette lignée est produite toute une série d'œuvres inspirées de manière plus ou moins directe de Boccace: Le Temple de Bocace de Georges Chastelain, le Portail du Temple de Bocace d'Antitus ou encore la Cité des Dames de Christine de Pizan ${ }^{28}$. Par ailleurs, vers 1401, est réalisée une traduction anonyme du De mulieribus claris de Boccace, qui connaît également un large succès. La pratique du catalogue de femmes illustres, dont Glenda Mc Leod a présenté un panorama depuis l'Antiquité ${ }^{29}$, est très vivante à cette époque et mérite qu'on s'y attarde. La

\footnotetext{
${ }^{26}$ Frédéric Duval, par exemple, dans son commentaire du Romuleon, essaie de mettre en évidence ces phénomènes d'interaction entre la France et l'Italie dans la diffusion de l'histoire romaine en France à la fin du Moyen Âge. Frédéric DuvAL, La Traduction $d u$ Romuleon par Sébastien Mamerot: étude sur la diffusion de l'histoire romaine en langue vernaculaire à la fin du Moyen Âge, Genève, Droz, 2001.

${ }^{27}$ Carla Bozzolo, Les Manuscrits des traductions françaises d'œuvres de Boccace, $X V^{e}$ siècle, Padoue, Editrice Antenore (Medioevo e umanesimo 15), 1973.

28 Jacqueline Cerquiglini-Toulet, «À la recherche des pères: la liste des auteurs illustres à la fin du Moyen Âge », MLN, vol. 116, n 4 (sept. 2001), p. 630-643. Voir aussi : «Fama et les Preux : nom et renom à la fin du Moyen Âge », Médiévales, 24 (printemps 1993), p. 35-44.

${ }^{29}$ Glenda MCLEOD, Virtue and Venom. Catalogs of Women from Antiquity to the Renaissance, Ann Arbor, The University of Michigan Press, 1991.
} 
critique s'est surtout intéressée au statut de la femme et aux rapports entre mauvaises femmes et femmes vertueuses qui se cristallisent autour de deux phénomènes: l'existence de catalogues stigmatisant les mauvaises conduites féminines depuis l'Antiquité et le choix du terme clarus et non illustris par Boccace, qui loin de faire un panégyrique de grandes figures féminines, met en évidence une notoriété qui doit souvent peu à la vertu. La comparaison des enjeux et des formes de ces différents textes permet de voir comment la transformation des objectifs entraîne une modification des conventions narratives ${ }^{30}$.

Les listes de personnages célèbres : une variation autour du thème des Hommes illustres?

Le goût médiéval des listes, longtemps considérées comme un « tic stylistique » de la période, a été examiné récemment par Madeleine Jeay qui lui a consacré un ouvrage entier ${ }^{31}$ où elle entend rectifier cette image en leur redonnant tout leur sens et en examinant leur fonction mémorielle. Quelles que soient 1'époque, la culture ou la langue, il existe des thèmes de prédilection de la liste, comme celles consacrées aux guerriers et aux héros, avec leurs modulations et altérations, depuis les pairs de Charlemagne jusqu'à l'évocation de figures exemplaires dans les romans. En quoi la liste des Hommes illustres se distingue de celles d'autres personnages célèbres ?

\footnotetext{
30 Rosalind Brown-Grant, in «Des hommes et des femmes illustres: modalités narratives et transformations génériques chez Pétrarque, Boccace et Christine de Pizan ", in Liliane Dulac et Bernard Ribemont (éd.), Une femme de lettres au Moyen Âge. Études autour de Christine de Pizan, Orléans, Paradigme (Medievalia, 16), 1995, p. 469-480, étudie, en comparant les préceptes et conventions suivis par Pétrarque, Boccace et Christine de Pizan, la façon dont cette dernière renouvelle la forme et les modalités narratives du catalogue de femmes illustres dans le Livre de la Cité des Dames pour promouvoir la vertu féminine, dans une problématique plus proche de celle de Pétrarque que de celle, contre-exemplaire, de Boccace.

31 Madeleine JEAY, Le Commerce des mots. L'usage des listes dans la littérature médiévale (XII $-X V^{e}$ siècle), Genève, Droz, 2006.
} 
Jacqueline Cerquiglini-Toulet a noté que fleurissent au $\mathrm{XIV}^{\mathrm{e}}$ siècle « des listes de noms qui sont des cristallisations de renom » et qui sont autant «d'indices montrant qu'une réflexion sur la gloire personnelle, le renom est en train de se mettre en place $»^{32}$. Émergent alors d'autres listes que celles des Hommes illustres dont les enjeux d'exemplarité et de glorification peuvent être comparables, qu'il s'agisse des Neuf Preux, des neuf muses, des listes d'amants malheureux, ou encore de celles qui relèvent de l'Ubi sunt. Jacqueline Cerquiglini-Toulet s'attache particulièrement aux listes d'écrivains célèbres. Les lettres et les arts deviennent source de renommée et elle examine comment s'est composé un canon littéraire, qui peut participer à l'illustration d'une ville ou d'une nation et dont l'énumération, dans la logique de succession et de filiation qu'elle crée, permet de « faire participer l'œuvre moderne à ce mouvement de la gloire». Ces phénomènes accompagnent «un mouvement de laïcisation » propre à l'époque: si Virgile conduisait Dante à travers l'Enfer et jusqu'aux portes du Paradis, la Sibylle guide Christine vers le Parnasse $^{33}$. Ainsi, la réflexion qui s'amorce en France autour de l'exemplarité et de la gloire se manifeste dans une pratique de la liste de grans hommes qui n'est pas sans rappeler par certains aspects celle qui se développe en Italie à la même époque autour des viris illustribus.

$\mathrm{Au}$ terme de ce parcours chronologique, il convient de souligner la richesse du thème des Hommes illustres. Elle s'exprime par la variété de ses supports (biographies, poésies, tapisseries, miniatures, statues, portraits et fresques) et par l'amplitude des permanences et mutations de son motif à travers le temps et surtout l'espace. La problématique centrale réside dans

\footnotetext{
32 Jacqueline Cerquiglini-Toulet, « À la recherche des pères : la liste des auteurs illustres à la fin du Moyen Âge », art. cit., p. 636.

${ }^{33}$ Ibid., p. 635.
} 
une tension qui s'exerce sur plusieurs plans: entre l'individuel et le collectif, entre les modèles de vie proposés et les modalités de la vie réelle du public, entre les différents usages politiques qui peuvent en être faits. Enfin se pose la question des productions aux marges du genre des Hommes illustres et des relations qu'elles entretiennent avec lui. Le débat sur sa circonscription n'est pas clos...

Daniel Ménager affirme qu'«imiter les grands hommes est le bréviaire de la Renaissance ${ }^{34}$, rappelant le succès de ce thème au $\mathrm{XVI}^{\mathrm{e}}$ siècle. Le bulletin de Questes s'est attaché à mettre en lumière non seulement les prémisses de cet engouement, mais encore les grands courants de pensée qui traversent la fin de cet âge qui n'a de moyen que le nom. Diachronie et synchronie doivent être mobilisées pour cerner au plus près ce que sont et qui sont les Hommes illustres. Il semble tout aussi important de souligner la permanence d'un genre depuis l'Antiquité. Le goût des références, des référents, sélectionnés et réinterprétés en fonction des besoins de la société ou de leurs élites politiques, semble être constant au moins jusqu'à la fin du XIX ${ }^{\mathrm{e}}$ siècle - la place emblématique du Panthéon n'étant pas l'exemple le moins fameux. Il nous faut alors découvrir le sens profond de cette continuité, au-delà des variations (de langue, de religion, de civilisation), et de cette réinvention permanente d'un genre à travers les siècles.

34 Daniel Menager, "Préface », in Patricia EICHel-Lojkine, Le Siècle des Grands Hommes. Les recueils de Vies d'hommes illustres avec portraits du XVI siècle, op. cit., p. 2. 